\title{
Processing and transmission of timing signals in synchronous networks
}

\author{
J.R.C. PIQUEIRA* and E.Y. TAKADA \\ Escola Politécnica da Universidade de São Paulo \\ Departamento de Engenharia de Telecomunicações e Controle \\ Av. Prof. Luciano Gualberto, travessa 3, n. 158 \\ 05508-900 São Paulo, SP, Brasil \\ E-mails: piqueira@lac.usp.br
}

\begin{abstract}
In order to have accurate operation, synchronous telecommunication networks need a reliable time basis signal extracted from the line data stream in each node. When the nodes are synchronized, routing and detection can be performed, guaranteeing the correct sequence of information distribution among the several users of a transmission trunk. Consequently, an auxiliary network is created inside the main network, a sub-network, dedicated to the distribution of the clock signals.

There are different solutions for the architecture of the time distribution sub-network and choosing one of them depends on cost, precision, reliability and operational security.

In this work we analyze the possible time distribution networks and formulate problems related to precision and stability of the timing signals by using the qualitative theory of differential equations. Correspondences between constitutive parameters of the networks and the dynamics of the spatial phase and frequency errors are established.
\end{abstract}

Mathematical subject classification: 70K20, 70K45.

Key words: bifurcation, master-slave network, phase-locked loop, synchronous network.

\section{Introduction}

The analysis of geographically separated oscillators started to become an important problem for telecommunications in the sixties with the introduction of the \#592/04. Received: 05/I/04. Accepted: 15/IV/04.

*Sponsored by CNPq of Brazil. 
first digital trunks which required synchronous time basis for demodulation and regeneration of pulse code modulation (PCM) signals [11, 14].

The phase-locked loop (PLL) is a device introduced by Belescize [3] in 1932 to extract timing signals. Nowadays, it is used in integrated circuit versions with high precision and low cost [4]. This device can extract the clock from digital signals corrupted by distortion and noise in transmission media. They were initially used in regenerators and termination units of digital multiplexing equipment $[19,17]$.

With the development of higher hierarchy multiplexing systems, mechanisms to guarantee synchronization between digital streams of lower hierarchy were required in the terminal stations. Then two different synchronization strategies were developed: PDH (Plesiochronous Digital Hierarchy) e SDH (Synchronous Digital Hierarchy) [2, 23].

Nodes in PDH systems operate with precise and independent clocks, corrected by operators from time to time. This is an expensive network as it requires precise oscillators in all nodes. With cheaper oscillators, the operational result is unsatisfactory implying bad performance.

In SDH systems, synchronization between nodes can be achieved with a few nodes with precise clocks. The others use PLLs for extracting the clock signal from the line with good precision and low cost.

In this work we are interested only in synchronous networks. We are going to discuss the several possible solutions for the architecture of the clock distribution network taking the dynamics of the PLL as the basis of our analysis.

The idea is to show that, in spite of the problem complexity, using Dynamical Systems theory is an interesting tool in order to obtain conditions for existence and stability of synchronous states.

\section{Phase-locking problem}

The problem of phase-locking consists of controlling the phase of a local oscillator by the phase of an external oscillation, making them coincide or, at least, differ by a constant. From the point of view of electronic engineering, PLL is the device that accomplishes it. It is a closed loop system connecting three basic elements: a phase detector (PD), a filter (F) and a voltage-controlled oscillator 
(VCO) $[4,10]$. A basic PLL is shown in figure 1.

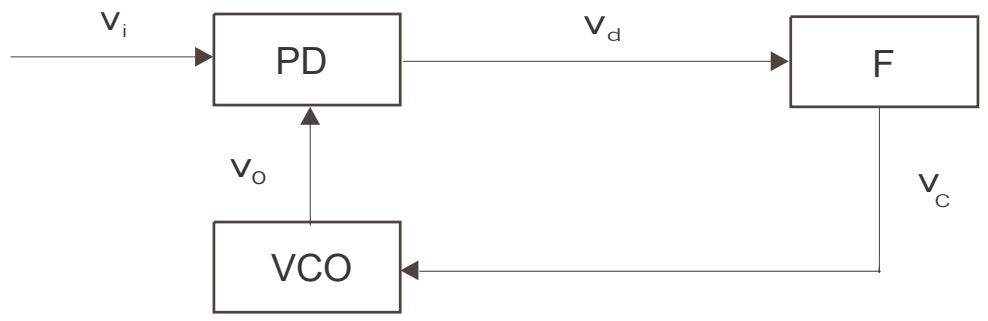

Figure 1 - Block diagram of a PLL.

The input and output signals are, respectively, given by:

$$
\begin{gathered}
v_{i}(t)=V_{i} \sin \left(\omega_{0} t+\theta_{i}(t)\right), \\
v_{0}(t)=V_{0} \cos \left(\omega_{0} t+\theta_{0}(t)\right)
\end{gathered}
$$

In these expressions, $\omega_{0}$ is the central frequency here named free-running frequency of the loop, $\theta_{i}(t)$ and $\theta_{0}(t)$ are the instantaneous phases, and $V_{i}$ and $V_{0}$ are the amplitudes of $v_{i}(t)$ and $v_{0}(t)$.

We consider the loop in a locked or synchronous state when it reaches an equilibrium state, with constant phase error $\varphi=\theta_{i}-\theta_{0}$ and null frequency error $\dot{\varphi}=\dot{\theta}_{i}-\dot{\theta}_{0}[4,10]$.

As the phase detector is a signal multiplier, the PD output is given by:

$$
v_{d}(t)=\frac{1}{2} K_{m} V_{i} V_{0}\left[\sin \left(\theta_{i}-\theta_{0}\right)+\sin \left(2 \omega_{0} t+\theta_{i}+\theta_{0}\right)\right],
$$

where $K_{m}$ is the phase detector gain.

The filter is supposed to eliminate high frequency terms. So, if the double frequency term is sufficiently attenuated by the filter [10], equation (1) is reduced to:

$$
v_{d}(t)=K_{d} \sin \left(\theta_{i}-\theta_{0}\right)
$$

with $K_{d}=\frac{1}{2} K_{m} V_{i} V_{0}$, in volts per radian.

Here we consider the model of PD and its output $v_{d}(t)$ given by equation (2). Being simple, we take the filter $\mathrm{F}$ as an all-pole low-pass with zeros in infinite 
$[20,5]$ and transfer function:

$$
F(s)=\frac{V_{c}(s)}{V_{d}(s)}=\frac{b_{0}}{s^{n}+b_{n-1} s^{n-1}+\cdots \cdots+b_{0}},
$$

where $V_{c}(s)$ and $V_{d}(s)$ represent the Laplace transforms of signals $v_{c}(t)$ and $v_{d}(t)$, respectively.

The combination of equations (2) and (3) yields:

$$
\frac{d^{n}}{d t^{n}} v_{c}(t)+b_{n-1} \frac{d^{n-1}}{d t^{n-1}} v_{c}(t)+\cdots+b_{0} v_{c}(t)=b_{0} K_{d} \sin \left(\theta_{i}-\theta_{0}\right) .
$$

The output phase of VCO $\theta_{0}$ is controlled by $v_{c}(t)$ and satisfies $\dot{\theta}_{0}=K_{0} v_{c}$, where $K_{0}$ is a VCO constant, in radians per volt per second [10]. Thus, equation (4) can be rewritten as:

$$
\begin{aligned}
& \frac{d^{n+1}}{d t^{n+1}} \theta_{0}(t)+b_{n-1} \frac{d^{n}}{d t^{n}} \theta_{0}(t)+\cdots \cdots+b_{0} \frac{d}{d t} \theta_{0}(t) \\
& =b_{0} K_{0} K_{d} \sin \left(\theta_{i}-\theta_{0}\right) .
\end{aligned}
$$

Defining $L(\cdot)$

$$
L(\cdot)=\frac{d^{n+1}}{d t^{n+1}}(\cdot)+b_{n-1} \frac{d^{n}}{d t^{n}}(\cdot)+\cdots+b_{0} \frac{d}{d t}(\cdot),
$$

and by taking the phase error $\varphi(t)=\theta_{i}-\theta_{0}$ as the dynamic variable, equation (5) becomes:

$$
L(\varphi)+b_{0} K_{0} K_{d} \sin \left(\theta_{i}-\theta_{0}\right)=L\left(\theta_{i}\right) .
$$

The ordinary differential equation (6) describes the behaviour of a PLL that is the main component of circuits for extracting time signals.

\section{Distribution of timing signals}

The problem of time distribution along networks consists of controlling frequency and phase of clock signals spreading over a wide area. The idea is synchronizing the frequency and phase scales of several oscillators in a network by using the data communication capacity of the links.

This problem has several applications [16]: 
- Establishing a world wide time distribution system;

- Synchronizing clocks located at different points in a digital communication network;

- Distributing time signals in a network in order to apply control actions and commands at specific times;

- Establishing a supercomputer by interconnecting several computers in a network.

These items are sufficient to justify the relevance of timing distribution in applications related to control and communication engineering.

In real problems, objective comparisons among the several possibilities are needed. Then, a precise mathematical treatment is necessary.

\subsection{Problem formulation}

As we have already stated, our intention is to discuss the several strategies for spreading clock signals and the synchronization of several oscillators distributed over a wide geographic area.

There are situations in which precision in synchronization is not a critical point. In these cases, independent clocks manually adjusted are used. This strategy originated the plesiochronous networks.

When synchronization results from interactions between the oscillators of the network we say that the network is synchronous.

Synchronous networks with a clock priority mechanism are called master-slave networks. When all the clocks in a network have equal relevance in determining the synchronous state, we say that the network is mutually synchronized.

Master-slave and mutually synchronized networks may include delay compensation techniques. Figure 2 shows a schematic diagram with the strategies of clock distribution [16].

In what follows, the phases of local oscillators, denoted by $\Phi$, are composed by a free-running term $\omega t$, a forcing term $\theta(t)$ and a perturbation $P(t)$, i.e.,

$$
\Phi(t)=\omega t+\theta(t)+P(t) .
$$




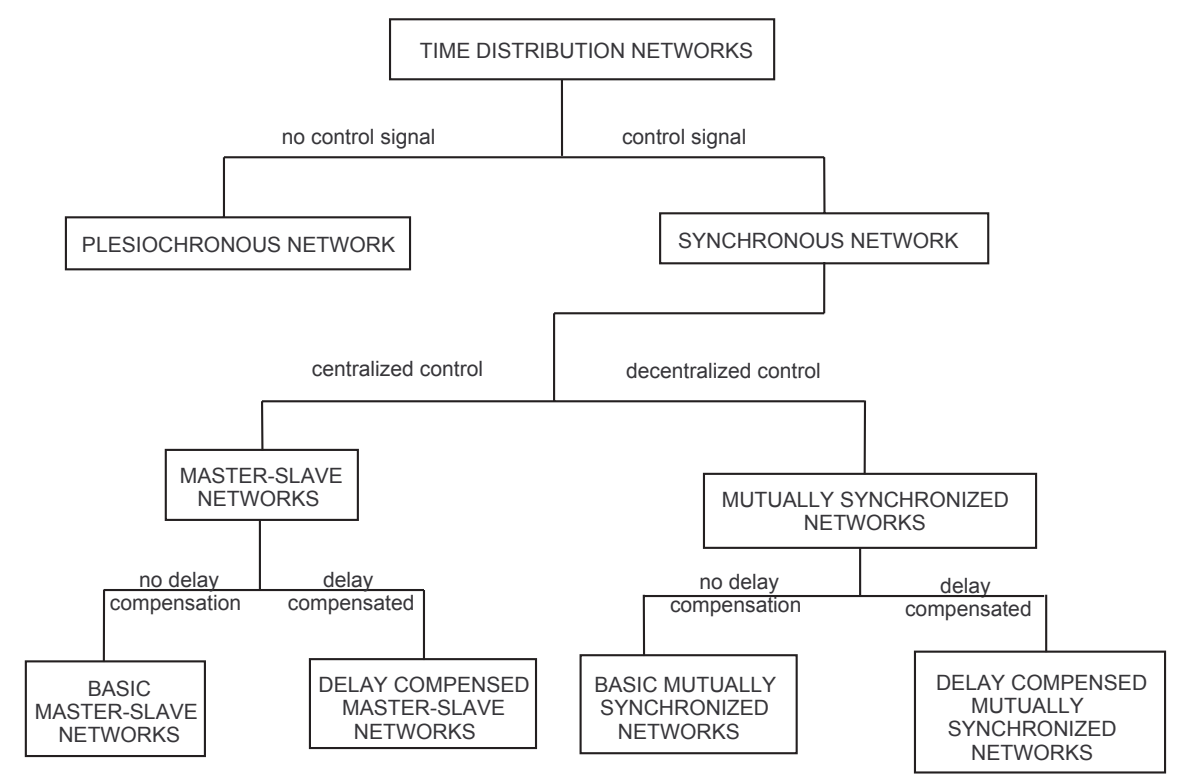

Figure 2 - Classification of clock signal distribution networks.

\subsection{Master-slave network classification}

Master-slave networks are classified according to the transmission direction of the time basis in One-Way Master-Slave (OWMS) and Two-Way Master-Slave (TWMS).

In OWMS networks, the master clock has its own and independent time basis. Slave clocks have their basis depending on a unique node, the master or another slave. Besides, these networks are classified according to the topology in chain and star.

In TWMS networks, the master clock has its own time basis but the control signal sent to the slave clocks is adjusted according to the basis of other nodes. Slave clocks may have their time basis dependent on several nodes.

According to the topology, TWMS networks can be classified as chain, star or loop.

The possible strategies for master-slave networks are detailed in figure 3.

Master-slave networks are extensively adopted in public telecommunication networks due to simple implementation, good timing performance, reliability, 
and low cost [6]. They also have applications in parallel distributed computation [24], robotics [15], and multimedia systems [25].

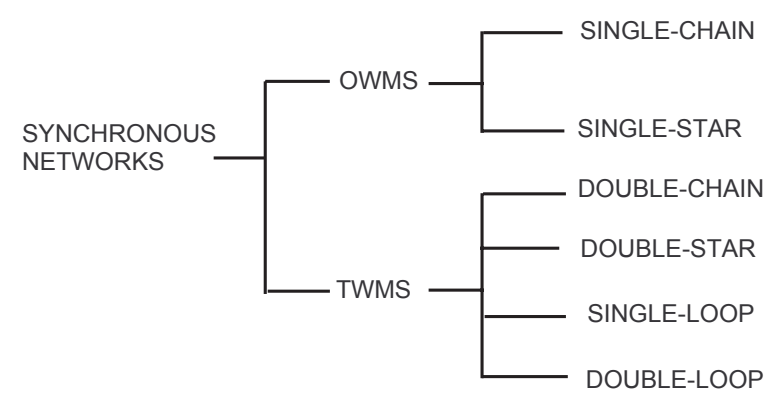

Figure 3 - Strategies for master-slave networks.

\subsubsection{Single-star OWMS network}

The topology of a single-star OWMS network is illustrated in figure 4. Masternode, denoted by M, sends its time basis for all other nodes.

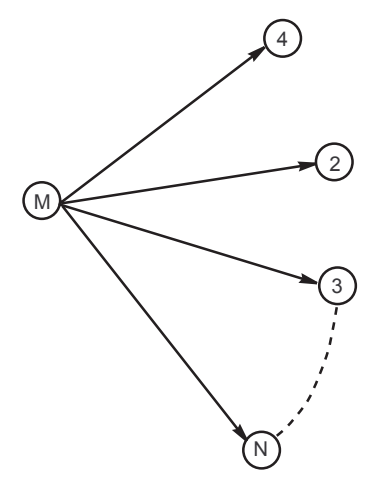

Figure 4 - Single-star OWMS network.

Master clock signal is independent on the other nodes. Its frequency is given by

$$
\dot{\Phi}_{1}=\omega_{M}+\Omega t,
$$

where $\omega_{M}$ is the frequency of normal operation of the master clock, and $\Omega t$ represents the deviation during the operation.

Slave-nodes are PLLs with input signal phase equal to the master-node phase, delayed by the propagation time from master to the considered slave node. 


\subsubsection{Single-chain OWMS network}

The topology of this network is shown in figure 5. The master clock, denoted by $\mathrm{M}$, sends its time basis to node- 2 , which sends its time basis to node-3, and so on, up to the last node.

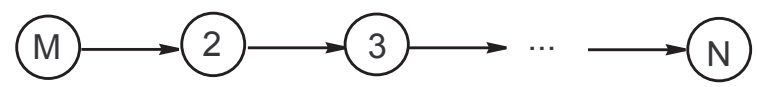

Figure 5 - Single-chain OWMS network.

Master-node, in this case, operates according to equation 7. Each slave clock can be considered a PLL.

As we have seen, the input signal phase in a node will be equal to the phase of the former node VCO, delayed by the propagation time.

\subsubsection{Double-star TWMS network}

This topology is illustrated in figure 6, with a master-node controlling the time basis of all slave nodes.

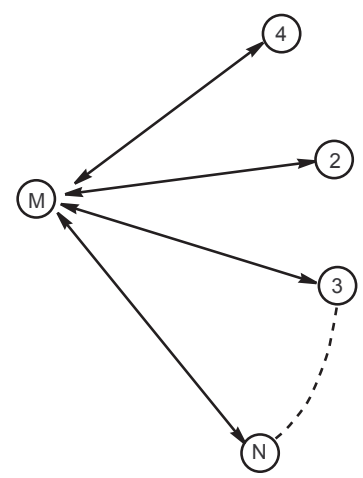

Figure 6 - Double-star TWMS network.

Master node $M$ has an accurate and independent time basis. However the control signal that it sends to the slaves considers its own phase and the phase of all slaves. 


\subsubsection{Double-chain TWMS network}

Figure 7 illustrates the topology of a double-chain TWMS network, with node $i-1$ working as a master for node $i$. When establishing its time basis, each slave uses the phase of its master and slave nodes.

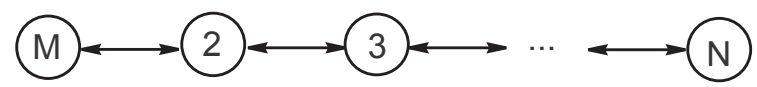

Figure 7 - Double-chain TWMS network.

The master node $M$ has an accurate and independent time basis. The control signal generated by the master $\mathrm{M}$ and sent to node 2 considers the phases of nodes $M$ and 2.

\subsubsection{Single-loop TWMS network}

In this topology, node $i-1$ works as a master for node $i$, as shown in figure 8 .

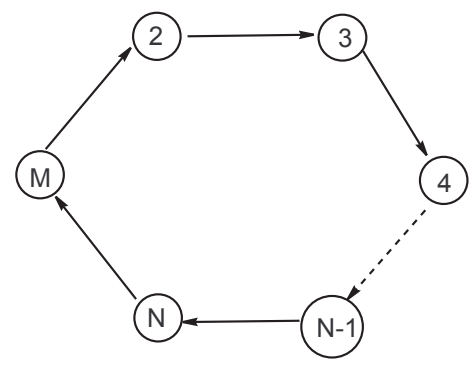

Figure 8 - Single-loop TWMS network.

The node $M$ has an accurate and independent time basis. The control signal generated by the master $M$ and sent to node 2, considers the phases obtained from $M$ and from slave $N$.

\subsubsection{Double-loop TWMS network}

The topology of this network is shown in figure 9, with node $i-1$ working as a master for node $i$. 
A slave-node establishes its time basis by considering the signals from its master and slave nodes.

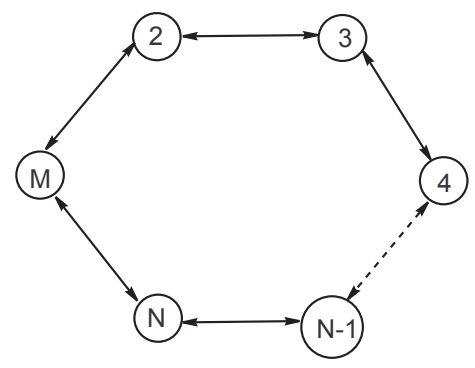

Figure 9 - Double-loop TWMS network.

The master node $M$ has an accurate and independent time basis. The control signal generated by the master $M$ and sent to nodes 2 and $N$, considers the phases obtained from $M$ and from slaves 2 and $N$.

\subsection{Master node in TWMS networks}

Figure 10 shows a scheme of master nodes in TWMS networks, indicating the mechanism for generating control signals considering the phase of the master $\Phi_{M}$ and the phase of the slaves $\Phi_{i}$.
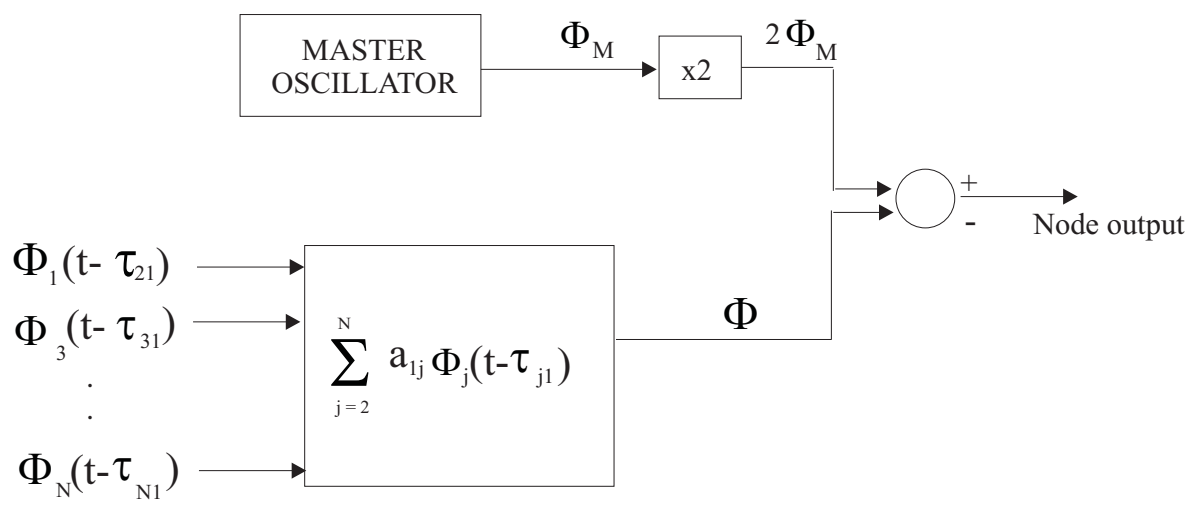

Figure 10 - Master-node in TWMS networks.

Control signals $\Phi$ sent by the master to the network is submitted to a weighting 
process that considers all the phase of the slaves with coefficients $a_{i, j}$ such that

$$
\sum_{j=2}^{N} a_{1, j}=1 .
$$

According to the network strategy the coefficients $a_{i, j}$ are:

double-chain: $\quad a_{1,2}=1$ and $a_{1, j}=0, \forall j \neq 2$

double-star: $\quad a_{1, j}=1 /(N-1), \forall j=2, \ldots, N$

simple-loop: $\quad a_{1, N}=1$ and $a_{1, j}=0, \forall j \neq N$

double-loop: $\quad a_{1,2}=a_{1, N}=1 / 2$ and $a_{1, j}=0, \forall j=3, \ldots, N-1$

\subsection{Slave node in TWMS networks}

In a TWMS network, PLL belonging to the i-slave node has an input signal with phase $\Phi$ resulting from a linear combination of phases from the several nodes, as shown in figure 11.

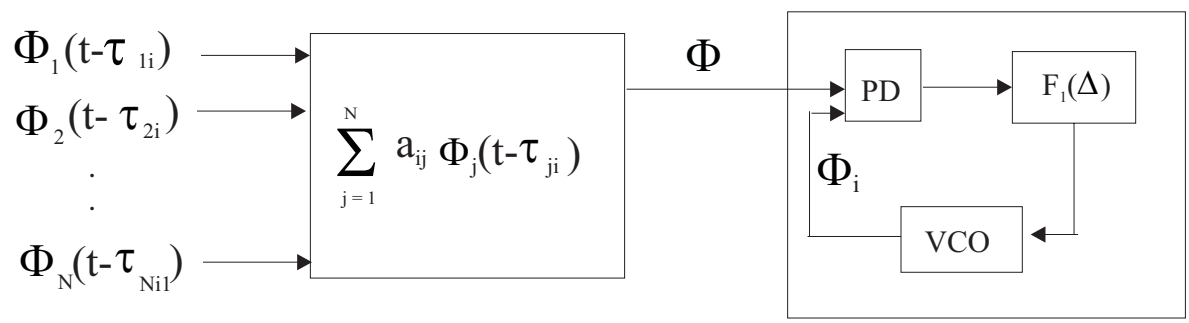

Figure 11 - Slave-nodes in TWMS networks.

The linear combination follows the condition

$$
\sum_{j=1, j \neq i}^{N} a_{i, j}=1,
$$

in each $i$ th-slave.

According to the clock distribution strategy, we have:

double-chain: $\quad a_{i, i+1}=a_{i, i-1}=1 / 2, \forall i=2, \ldots, N-1$ and

$$
a_{N, 1}=a_{N, N-1}=1 / 2
$$

double-star: $\quad a_{i, 1}=1$ and $a_{i, j}=0, \forall i, j=2, \ldots, N$

simple-loop: $\quad a_{i, i-1}=1$

double-loop: same as double chain 


\section{Center Manifold Theorem applied to the comparison between OWMS topologies}

As explained in the last section, there are a lot of possible solutions regarding to the architecture of a time distribution network topology.

When designing the network it is important to know the robustness of the synchronous state depending on the constitutive parameters of the nodes.

Reasoning in this way, the Center Manifold Theorem from Dynamical Systems Theory is useful to study the behaviour of solutions near equilibrium states providing important hints about the synchronous state stability.

As an example, in this section we compare two OWMS topologies, single-star and single-chain, by applying the Center Manifold Theorem [18].

The nodes of the network are supposed to have second order PLLs as timing detectors. Slow instabilities and Doppler effect of master signal are neglected in our modeling.

\subsection{Single-star OWMS network}

The network to be analyzed consists of a master and two slaves in a single-star OWMS network.

Node 1 is the master with a free oscillator with phase $\Phi_{1}(t)$. Nodes 2 and 3 are the slaves and they are second order PLLs with $\mu_{1}$ being the filter time constant and $\mu_{2}$, the loop gain.

Phases of slaves, $\Phi_{2}(t)$ and $\Phi_{3}(t)$, respectively, are controlled by the master phase signal. Transmission delays $\tau_{i, 1}$ from master node to $i$ th-node, for simplicity, will be considered equal $\tau_{2,1}=\tau_{3,1}=\tau$.

Then, the network dynamics is given by:

$$
\begin{aligned}
\ddot{\varphi}_{i j}+\mu_{1} \dot{\varphi}_{i j}+\mu_{2} \operatorname{sen}\left(\varphi_{i i}\right) & =\ddot{\varphi}_{1}+\mu_{1} \dot{\varphi}_{1}, \\
\ddot{\varphi}_{i i}+\mu_{1} \dot{\varphi}_{i i}+\mu_{2} \sin \left(\varphi_{i i}\right) & =\ddot{\varphi}_{1}\left(t-\tau_{i j}\right)+\mu_{1} \dot{\varphi}_{1}\left(t-\tau_{i j}\right) .
\end{aligned}
$$

The subscript $j$ stands for the master, and $i$ for the slaves. Consequently, $\varphi_{i i}$ is the difference between the phase of the VCO output signal and the phase of the PD input signal in the $i$ th-slave and is called local phase error.

In order to represent the spatial phase error we use $\varphi_{i j}$ that is the difference between the phase of the VCO output signal of node $i$ and the phase of the master. 
By considering that the phase of the master output signal is a step at $\mathrm{t}=0$, we have: $\dot{\Phi}_{1}(t)=\dot{\Phi}_{1}(t-\tau)=0$ and $\ddot{\Phi}_{1}(t)=\ddot{\Phi}_{1}(t-\tau)=0$, considering $t \geq \tau$.

Re-scaling the time variable $T=\mu_{1} t$, equation (8) becomes:

$$
\begin{aligned}
& \varphi_{21}^{\prime \prime}+\varphi_{21}^{\prime}+\mu \sin \varphi_{22}=0, \\
& \varphi_{22}^{\prime \prime}+\varphi_{22}^{\prime}+\mu \sin \varphi_{22}=0, \\
& \varphi_{31}^{\prime \prime}+\varphi_{31}^{\prime}+\mu \sin \varphi_{33}=0, \\
& \varphi_{33}^{\prime \prime}+\varphi_{33}^{\prime}+\mu \sin \varphi_{33}=0,
\end{aligned}
$$

where $\mu=\mu_{2} / \mu_{1}^{2}$ and $x^{\prime}=d x / d T$.

Equations (9) show that the dynamics of the interaction between nodes 1 and 2 and between nodes 1 and 3 are identical and there are two pairs of non coupled differential equations.

Without loss of generality, we are going to study the equations related only to the first pair of nodes.

By choosing the state variables as $x_{1}=\varphi_{21}^{\prime}, x_{2}=\varphi_{22}$ and $x_{3}=\varphi_{22}^{\prime}$ :

$$
\left\{\begin{array}{l}
x_{1}^{\prime}=-x_{1}-\mu \sin x_{2}, \\
x_{2}^{\prime}=x_{3}, \\
x_{3}^{\prime}=-x_{3}-\mu \sin x_{2} .
\end{array}\right.
$$

This system admits a cylindrical phase surface, so equilibrium states of (10) are $P_{1}=(0,0,0)$ e $P_{2}=(0,-\pi, 0)$, and the eigenvalues of the Jacobian matrix associated, calculated in $P_{1}$, are:

$$
\lambda_{1}=-1, \lambda_{2}=\frac{-1-\sqrt{1-4 \mu}}{2} \text { and } \lambda_{3}=\frac{-1+\sqrt{1-4 \mu}}{2} .
$$

By observing the eigenvalues, we conclude that:

- for $\mu>0: \operatorname{dim}\left(E^{s}\right)=3, P_{1}$ is asymptotically stable.

- for $\mu<0$ : $\operatorname{dim}\left(E^{s}\right)=2$ and $\operatorname{dim}\left(E^{u}\right)=1, P_{1}$ is unstable.

- for $\mu=0: \operatorname{dim}\left(E^{s}\right)=2, \operatorname{dim}\left(E^{c}\right)=1$, and nothing can be said about $P_{1}$ stability observing only the eigenvalues of the Jacobian matrix. 
Analogously, the eigenvalues of Jacobian matrix in $P_{2}$ are:

$$
\lambda_{1}=-1, \lambda_{2}=\frac{-1-\sqrt{1+4 \mu}}{2} \text { and } \lambda_{3}=\frac{-1+\sqrt{1+4 \mu}}{2} .
$$

Therefore:

- for $\mu<0$ : $\operatorname{dim}\left(E^{s}\right)=3, P_{2}$ is asymptotically stable.

- for $\mu>0$ : $\operatorname{dim}\left(E^{s}\right)=2$ and $\operatorname{dim}\left(E^{u}\right)=1, P_{2}$ is unstable.

- for $\mu=0: \operatorname{dim}\left(E^{s}\right)=2$ and $\operatorname{dim}\left(E^{c}\right)=1$, nothing can be said about $P_{2}$ stability observing only the eigenvalues of the Jacobian matrix.

When $\mu=0$ the stability of $P_{1}$ and $P_{2}$ changes, i.e., there is a bifurcation.

In this case, we have to analyze how the system behavior depends on $\mu$, restricted to its central manifold. In order to do this, we rewrite the equations (10) including the parameter in the dynamics [27].

$$
\left\{\begin{array}{l}
x_{1}^{\prime}=-x_{1}-\mu \sin x_{2} \\
x_{2}^{\prime}=x_{3} \\
x_{3}^{\prime}=-x_{3}-\mu \sin x_{2} \\
\mu^{\prime}=0
\end{array}\right.
$$

The eigenvalues of the Jacobian matrix associated to system (11) calculated in $\left(x_{1}, x_{2}, x_{3}, \mu\right)=(0,0,0,0)$ are $\lambda_{1}=-1, \lambda_{2}=-1$ and $\lambda_{3}=0$.

By using the Taylor approximation $\sin x_{2}=x_{2}-\frac{x_{2}^{3}}{6}+\mathcal{O}\left(x_{2}^{5}\right)$ and Jordan canonical form, we have:

$$
\left[\begin{array}{l}
v_{1}^{\prime} \\
v_{2}^{\prime} \\
v_{3}^{\prime}
\end{array}\right]=\left[\begin{array}{rrr}
-1 & 0 & 0 \\
0 & -1 & 0 \\
0 & 0 & 0
\end{array}\right]\left[\begin{array}{l}
v_{1} \\
v_{2} \\
v_{3}
\end{array}\right]+\left[\begin{array}{l}
g_{1} \\
g_{2} \\
f
\end{array}\right],
$$

with

$$
f=g_{1}=g_{2}=\mu\left[v_{1}-\frac{v_{1}^{3}}{6}-v_{3}+\frac{v_{1}^{2} v_{3}}{2}-\frac{v_{1} v_{3}^{2}}{2}+\frac{v_{3}^{3}}{6}\right] .
$$

So, from Central Manifold Theorem, the stability of $(0,0,0)$ near $\mu=0$ can be determined by analyzing the vector field restricted to a central manifold 
$W^{c}(0), 0 \in \mathbb{R}^{3}$. In our case, we can write:

$$
\begin{aligned}
W^{c}(0)=\{ & \left(v_{1}, v_{2}, v_{3}, \mu\right) \in \mathbb{R}^{4} / v_{1}=h_{1}(x, \mu), v_{2}=h_{2}(x, \mu), v_{3}=x, \\
& \left.h_{i}(0,0)=0, D h_{i}(0,0)=0, i=1,2\right\}
\end{aligned}
$$

for $x$ and $\mu$ sufficiently small.

By applying the center manifold theorem [7,27], and considering polynomial approximations, we have:

$$
h_{1}(x, \mu)=\frac{\mu}{6} x^{3} \text { and } h_{2}(x, \mu)=\frac{\mu}{6} x^{3} .
$$

Replacing (13) in the third equation of (12) and by considering the forth order terms, we have the vector field, reduced to the center manifold $W^{c}(0)$, giving by:

$$
\left\{\begin{array}{l}
x^{\prime}=\frac{\mu(\mu-1)}{6} x^{3}-\mu x \\
\mu^{\prime}=0 .
\end{array}\right.
$$

When we plot the equilibrium states of (14) we can observe from the bifurcation diagram (figure 12) that $x=0$ is a stable equilibrium state for $\mu>0$ and unstable for $\mu<0$. When $\mu>1$, two new unstable equilibrium states given by $x^{2}=1 /(\mu-1)$ are created.

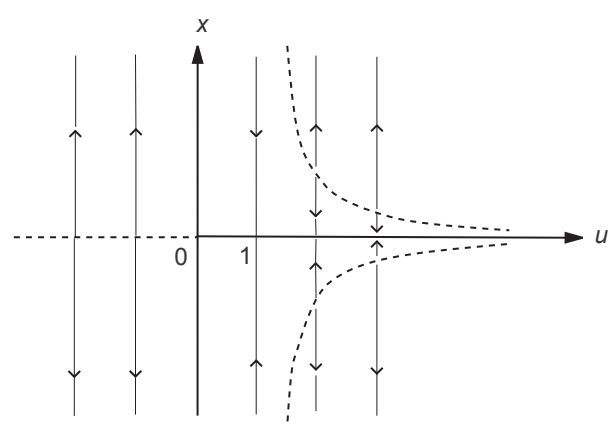

Figure 12 - Bifurcation diagram for $P_{1}$.

An analogous reasoning can be conducted for $P_{2}$, and its stability near $\mu=0$ can be studied by analyzing the bifurcation diagram shown in (figure 13). 


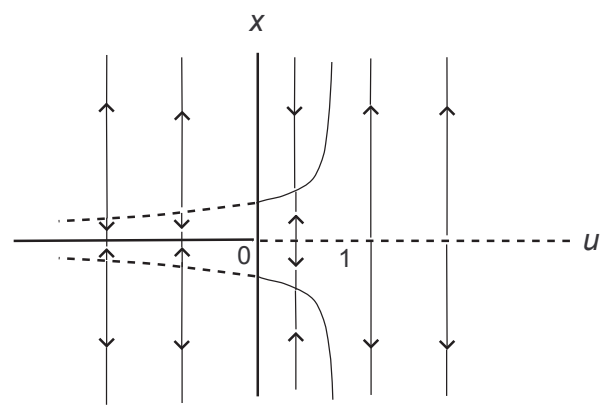

Figure 13 - Bifurcation diagram for $P_{2}$.

\subsection{Single-chain OWMS network}

The dynamics of a network consisting of a master and two slaves in a single-chain topology is just the same as the considered in equation 8 , with $\Phi_{1}(t)$ being the phase of the master. $\Phi_{2}(t)$ and $\Phi_{3}(t)$ are the phases of the slaves.

Changing the time scale by $T=\mu_{1} t$, and considering a step with finite amplitude as the output signal of master, the dynamics of a single-chain OWMS network is given by:

$$
\begin{aligned}
& \varphi_{21}^{\prime \prime}+\varphi_{21}^{\prime}+\mu \operatorname{sen} \varphi_{22}=0, \\
& \varphi_{22}^{\prime \prime}+\varphi_{22}^{\prime}+\mu \operatorname{sen} \varphi_{22}=0, \\
& \varphi_{32}^{\prime \prime}+\varphi_{32}^{\prime}+\mu \operatorname{sen} \varphi_{33}=0, \\
& \varphi_{33}^{\prime \prime}+\varphi_{33}^{\prime}+\mu \operatorname{sen} \varphi_{33}=0 .
\end{aligned}
$$

With these equations, we conclude that the dynamics of a single-chain network and of a single-star network, submitted to step inputs, are the same because these networks are described by the same equations.

\subsection{Engineering conclusion}

By using the Center Manifold Theory we might formulate the problem of the dynamics of single-chain and single-star OWMS networks and useful engineering conclusions could be:

- Delays are irrelevant when we consider a step with finite amplitude as the input in our problem. 
- For a given phase and frequency initial state, parameter $\mu$ that determines the existence and stability [13] of the synchronous state changes the dynamics as illustrated in bifurcation diagrams shown in figures 12 and 13.

\section{Stability of equilibrium states and frequency errors in a double-star TWMS network}

When a TWMS strategy of clock distribution is chosen we have a more robust and accurate performance for the network. But, in this situation, due to the feedback loops between the nodes, frequency errors, even low, propagate along the whole network spoiling the performance.

In this section, we study the problem of frequency error propagation in doublestar TWMS networks by using techniques from dynamical systems theory [22] obtaining conditions for existence and stability of synchronous states [13].

The slaves considered are second order PLLs with a time constant $\mu$. The architecture is the double-star with a master $M$ and $N-1$ slaves.

The master is an oscillator with phase $\Phi_{M}(t)$. Signal propagation time from the master to the $i$ th slave is indicated by $\tau_{1 i}$, and, from the $i$ th slave to the master, by $\tau_{i 1}$, for $i=2, \ldots, N$.

Phases of oscillators output in this network are defined as follows:

- Master oscillator

$$
\Phi_{M}(t)=\omega_{M} t+P_{M}(t)
$$

- $i$ th slave-node oscillator

$$
\Phi_{i}(t)=\theta_{i}(t)+\omega_{i} t+P_{i}(t), \quad i=2,3,4, \ldots, N .
$$

- Master output phase

$$
\Phi_{1}(t)=2 \Phi_{M}(t)-\frac{1}{N-1} \sum_{i=2}^{N} \Phi_{i}\left(t-\tau_{i 1}\right) .
$$


Modeling each $i$ th slave, $i=2,3,4, \ldots, N$, with a PLL equation, as we have seen in section 2 , the dynamics can be described as follows:

$$
\begin{gathered}
\ddot{\phi}_{i}(t)+\mu \dot{\phi}_{i}(t)-\mu \mu_{i} \operatorname{sen}\left(\phi_{1}\left(t-\tau_{1 i}\right)-\phi_{i}(t)\right) \\
=\ddot{P}_{i}(t)+\mu \omega_{i}+\mu \dot{P}_{i}(t),
\end{gathered}
$$

where $\mu$ is the filter cut-off frequency in all nodes, and $\mu_{i}$ is the $i$ th slave-node PLL gain.

Defining frequency and phase spatial errors by:

$$
\left\{\begin{array}{l}
\varphi_{M, i}=\phi_{M}-\phi_{i}, \\
\dot{\varphi}_{M, i}=\dot{\phi}_{M}-\dot{\phi}_{i} .
\end{array}\right.
$$

Considering phase perturbations of second order with master acceleration $\Omega_{M}$ and slave acceleration $\Omega_{i}$, the substitution of equations (16), (17) and (18) in (19), taking into account equation (20), results:

$$
\begin{gathered}
\ddot{\varphi}_{M i}+\mu \dot{\varphi}_{M i}+\mu \mu_{i} \sin \left[\frac{N}{N-1} \varphi_{M i}+\frac{1}{N-1} \sum_{\substack{j=2 \\
j \neq i}}^{N} \varphi_{M, j}\right. \\
\left.-\frac{1}{N-1} \sum_{j=2}^{N}\left(\tau_{1 i}+\tau_{j 1}\right) \dot{\varphi}_{M j}-\frac{1}{N-1}\left[(N-1) \tau_{1 i}+\sum_{j=2}^{N} \tau_{j 1}\right]\left(\omega_{M}+\Omega_{M} t\right)\right] \\
=-\Omega_{i}-\mu \Omega_{i} t-\mu \omega_{i}+\Omega_{M}+\mu \Omega_{M} t .
\end{gathered}
$$

The dynamics is non-linear and depends explicitly on time, so there is no equilibrium state.

Consequently, the oscillator degradation combined with the delays does not allow the system to be locked in the steady state $[9,21]$.

If we take the derivatives in equation (21) and consider a linear approximation by expanding the non-linear terms in Taylor series [10,9,21], we have:

$$
\begin{gathered}
\dddot{\varphi}_{M i}+\mu \ddot{\varphi}_{M i}+\mu \mu_{i}\left[\frac{N}{N-1} \dot{\varphi}_{M i}+\frac{1}{N-1} \sum_{\substack{j=2 \\
j \neq i}}^{N} \dot{\varphi}_{M, j}\right. \\
\left.-\frac{1}{N-1} \sum_{j=2}^{N}\left(\tau_{1 i}+\tau_{j 1}\right) \ddot{\varphi}_{M j}-\frac{1}{N-1}\left[(N-1) \tau_{1 i}+\sum_{j=2}^{N} \tau_{j 1}\right] \Omega_{M}\right] \\
=\mu\left(\Omega_{M}-\Omega_{i}\right) .
\end{gathered}
$$


Considering the state variables:

$$
x_{2 i-3}=\dot{\varphi}_{M, i} \quad \text { and } \quad x_{2 i-2}=\ddot{\varphi}_{M, i}
$$

the system becomes:

$$
\left\{\begin{aligned}
\dot{x}_{2 i-3}= & x_{2 i-2}, \\
\dot{x}_{2 i-2}= & -\mu x_{2 i-2}-\mu \mu_{i}\left[\frac{1}{N-1} \sum_{\substack{j=2 \\
j \neq i}}^{N-2} x_{2 j-3}+\frac{N}{N-1} x_{2 i-3}\right. \\
& -\frac{1}{N} \sum_{j=2}^{N}\left(\tau_{1 i}+\tau_{j 1}\right) x_{2 j-2}-\frac{1}{N-1}\left[(N-1) \tau_{1 i}\right. \\
& \left.\left.-\sum_{j=2}^{N} \tau_{j 1}\right] \Omega_{M}\right]+\mu\left(\Omega_{M}-\Omega_{i}\right) .
\end{aligned}\right.
$$

This system admits an equilibrium state which corresponds to constant frequency spatial errors $\dot{\varphi}_{M, i}$ and non-limited phase spatial errors $\varphi_{M, i}$.

Acceleration spatial error $\ddot{\varphi}_{M, i}$ tends to a zero stationary state. After the transient states, the acceleration of any slave follows the acceleration of the master.

The linear part of the new system, around the equilibrium state, can be represented by:

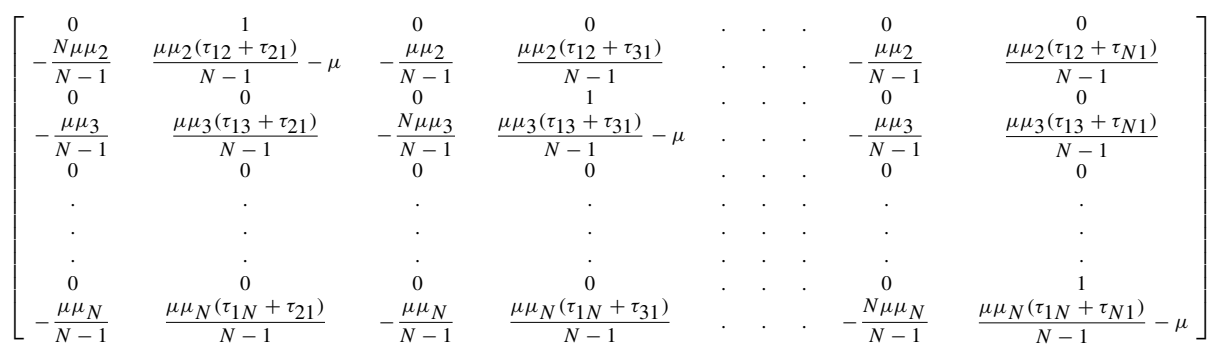

If we assume the simplifying but realist fact that delays are close to the gain of the PLLs, with

$$
\tau_{1 i}=\tau_{i 1}=\tau, \quad \mu_{i}=v \quad \text { and } \quad \Omega_{i}=\Omega, \quad i=2,3, \ldots, N,
$$


the equilibrium state is given by:

$$
\sum_{\substack{j=2 \\ j \neq i}}^{N-2} x_{2 j-3}+N x_{2 i-3}=\frac{N-1}{2 v}\left(\Omega_{M}-\Omega\right) \quad \text { and } \quad x_{2 i-2}=0 .
$$

Developing the expressions and verifying the influence of the number of nodes, we can write the equilibrium state by using mathematical induction as:

$$
x_{2 i-3}=\frac{1}{2 v}\left(\Omega_{M}-\Omega\right) \quad \text { and } \quad x_{2 i-2}=0, \quad i=2,3, \ldots, N .
$$

The linear part of this new system around the equilibrium state is represented by matrix $A$ :

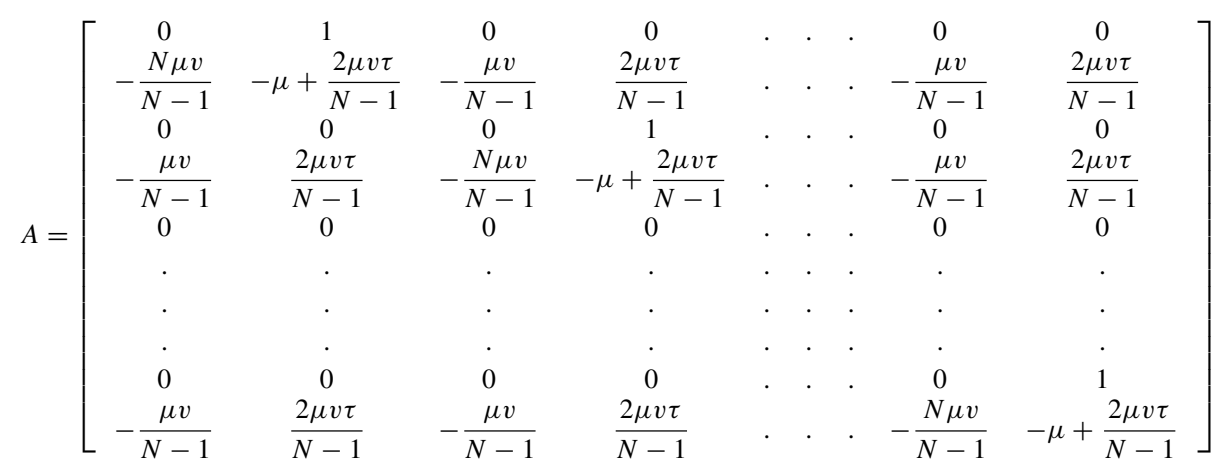

Calculating the eigenvalues of $A$ by using MAPLE V [1]:

$$
\begin{aligned}
& \lambda_{1}=\lambda_{2}=\ldots=\lambda_{r}=-\frac{\mu}{2}+\sqrt{\mu^{2}-4 \mu v}, \\
& \lambda_{r+1}=\lambda_{r+2}=\ldots=\lambda_{2(N-2)}=-\frac{\mu}{2}-\sqrt{\mu^{2}-4 \mu v}, \\
& \lambda_{2(N-1)-1,2(N-1)}=\mu v \tau-\frac{\mu}{2} \pm \sqrt{\left(\mu v \tau-\frac{\mu}{2}\right)^{2}-2 \mu v .}
\end{aligned}
$$

Examining the eigenvalues we observe that the equilibrium point of the system, having constant acceleration error, is asymptotically stable for any physical possible value of the parameters. 


\subsection{Another engineering conclusion}

As a result of long-term instability of the master oscillator in a double star TWMS network, slave nodes do not synchronize in phase with master. Phase spatial error $\varphi_{M, i}$ between a slave and the master is unlimited as a consequence of equation (21).

However, in some practical situations, we can relate the propagation times with the gains of PLLs making the frequency errors controllable.

Summarizing:

- It follows from equation (21) that the double-star TWMS network does not present synchronous solution when oscillators suffer a phase acceleration and frequency errors propagate along the whole network.

- It follows from (25) that the frequency spatial errors do not dependent on the number of slaves but only on the acceleration of the master and the slaves.

- Restrictions on the stability domain of equilibrium state depend on the relation between PLLs gains and signal delays.

\section{Final comment}

The problem of choosing a good circuit for time distribution networks has many aspects mainly related to topology and parameter design.

Modeling the several possible networks gives a nonlinear high dimension ordinary differential equation and analytical solutions are hard to be obtained.

Using Dynamical System Theory is a very useful tool for this kind of problem providing existence and stability conditions for the synchronous state of the networks, relating circuit parameters, transmission delays and deviations.

\section{REFERENCES}

[1] M.L. Abell and J.P. Braselton, “Maple V by example”, Academic Press, London, (1999).

[2] J.C. Bellamy, Digital network synchronization, IEEE Communications Magazine (1995), $70-83$.

[3] H. de Bellescize, La reception synchrone, Onde Electrique, 11 (1932), 230-240. 
[4] R.E. Best, “Phase-locked loops $-4^{\text {th }}$ edition”, McGraw Hill, New York, (1999).

[5] H.J. Blinchkoff, All-Pole Phase-Locked Tracking Filters, IEEE Trans. Commun., vol COM30, pp. 2312-2318, Oct. 1982.

[6] C.S. Bregni, A historical perspective on telecomunnications network synchronization, IEEE Commun. Magazine, pp. 158-166, June 1998.

[7] J. Carr, “Applications of Centre Manifold Theory’, Springer, New York, (1981).

[8] R.D. Cideciyan and W.C. Lindsey, Effects of Long-Term Clock Instability on Master-Slave Networks, IEEE Trans. Commun., vol COM-35, pp. 950-955, Sep. 1987.

[9] P.A. Garcia, Redes simples de malhas de sincronismo de fase: uma análise via teoria de sistemas dinâmicos, Dissertaçãode Mestrado, Universidade Mackenzie, São Paulo, 2000.

[10] F.M. Gardner, “'Phase-lock Techniques”, John Wiley \& Sons, New York, (1979).

[11] A. Gersho and B.J. Karafin, Mutual Synchronization of Geographically Separeted Oscillators, The Bell System Technical Journal (1966), 1689-1704.

[12] J. Guckenheimer and P. Holmes, “Nonlinear Oscillations, Dynamical Systems and Bifurcation of Vector Fields $5^{\text {th }}$ edition", Springer, New York, (1997).

[13] T. Kailath, “'Linear Systems”, Prentice-Hall, New Jersey, (1980).

[14] M. Karnaugh, A Model for Organic Synchronization of Communications Systems, The Bell System Technical Journal (1966), 1705-1735

[15] W.C. Lee, J. Lee, D. Choi, M. Kim and C. Lee, The Distributted Controller Architecture for a Masterarm and its Application to Teleoperation with Force Feedback, IEEE International Conference on Robotics and Automation, pp. 213-218, (1999).

[16] W.C. Lindsey, F. Ghazvinian, W.C. Hagmann and K. Dessouky, Network Synchronization, Proceedings of the IEEE, vol 73, 1445-1467, Oct. 1985.

[17] W.C. Lindsey and M.K. Simon, “Telecommunication Systems Engineering', Dover, New York, (1973).

[18] C.N. Marmo, V.F. de Faria, L.H.A. Monteiro e J.R.C. Piqueira, Sincronismo em Redes Mestre-Escravo: comparação de topologias, XIV Congresso Brasileiro de Automática, NatalRN-Brasil, 2002, pp 691-696.

[19] M. Schwartz, “Transmissão de Informação, Modulação e Ruído”, Guanabara Dois, Rio de Janeiro, 1979 (translated from the edition: McGraw Hill, New York, 1970).

[20] K. Ogata, “'Modern Control Engineering’, Prentice Hall, New Jersey, (1997).

[21] J.R.C. Piqueira, Uma contribuição ao estudo das redes com malhas de sincronismo de fase, Tese de Livre Docência, EPUSP, São Paulo, (1997).

[22] S.A. Castillo-Vargas, Propagação de erros de frequiência em redes mestre-escravo, Dissertação de Mestrado, EPUSP, São Paulo, (2002). 
[23] M. Sexton and A. Reid, “Transmission Networking: SONET and Synchronous Digital Hierarchy”, Norwood, MA, Artech House, (1992).

[24] G. Shao, F. Berman and R. Wolski, Master/slave Computing on the Grid, Proceedings of the IEEE COMPSAC, 2000.

[25] S. Sohail and G. Raj, Replication of Multimedia Data using Master-Slave architecture, Proceedings of the IEEE $21^{\text {st }}$ COMPSAC, (1997).

[26] A. Weinberg and B. Liu, Discrete Time Analysis of Non-uniform Sampling: First and Second Order Digital Phase-Locked Loops, IEEE Trans. Commun., vol COM-22, pp. 123-137, Feb. 1974.

[27] S. Wiggins, “'Introduction to Applied Nonlinear Dynamical Systems and Chaos", Springer, New York, (1990). 